\title{
Quantum Limits for Measurements on Macroscopic Bodies : a Decoherence Analysis
}

\author{
Carlos O. Escobar, Léa Ferreira dos Santos \\ and \\ Paulo C. Marques * \\ Instituto de Física da Universidade de São Paulo \\ 01452-990 C.P. 20516-São Paulo, SP \\ Brazil
}

\begin{abstract}
We consider in this paper the quantum limits for measurements on macroscopic bodies which are obtained in a novel way employing the concept of decoherence coming from an analysis of the quantum mechanics of dissipative systems. Two cases are analysed, the free mass and the harmonic oscillator, and for both systems we compare our approach with previous treatments of such limits.
\end{abstract}

03.65.Bz , 04.60.+n

Typeset using REVTEX

*e-mail : pacmarques@uspif.if.usp.br 


\section{INTRODUCTION}

One of the outstanding problems in contemporary physics is the detection of the gravitational waves predicted by Einstein in his General Relativity Theory [1]. The detection of such waves presents a challenge to the experimentalists who have to monitor extremely small fluctuations in the position of macroscopic bodies [2]. The sensitivity planned for the future generation of detectors, will bring us close to the limits imposed by quantum mechanics [3].

It is our purpose in this paper to examine in detail the determination of quantum limits for free masses and for the harmonic oscillator, subjected to thermal fluctuations. We rely on the methods developed by several authors to analyse the quantum mechanics of a system in interaction with its environment 4 .

We differ in this respect from Braginsky [3] who uses a two step procedure in order to obtain the quantum limits, firstly considering the limits imposed by the uncertainty principle on an isolated system, not subjected to thermal fluctuations and then in the second step looking at the same system, this time behaving classically but interacting with the environment.

This procedure neglects the fact that the system is always in contact with the environment. We remove this asymmetry treating the system quantum mechanically and subjected to thermal fluctuations from the very beginning.

We want to investigate the conditions under which the quantum behavior of a macroscopic body cannot be ignored or equivalently for our problem, when classical behavior sets in. This problem has become of importance in recent studies of quantum cosmology [5] as well as in the quantum theory of the measurement process [6]. The concept of decoherence arising from these studies will be central in our considerations. The way we see the onset of quantum behavior of a macroscopic body is related to the decoherence time scale in the following way : A macroscopic test body must be treated quantum mechanically if the time interval during which a measurement of some of its observables is made, is smaller than the decoherence time. 
In section II we briefly review the concept of decoherence as used by Zurek and collaborators [7]. In section III we use the decoherence time scale in order to obtain the conditions for quantum behavior of a macroscopic body in two cases : the harmonic oscillator and the free mass (free in the sense it is not subjected to an external field but, of course, in contact with a reservoir). We end section III with a criticism of Braginsky's derivation of the quantum condition for a free mass. In section IV we conclude the paper outlining some future and open problems.

\section{DECOHERENCE TIME SCALE}

When a system interacts with a thermal reservoir, it is usual to analyse its evolution in terms of the reduced density matrix $\left(\rho_{r}\right)$, obtained by coarse graining from the full density matrix $(\rho)$ for the system plus reservoir. This coarse graining is effected by tracing out the degrees of freedom of the environment :

$$
\rho_{r}=\operatorname{Tr}_{E}[\rho]
$$

where $E$ refers to the environment variables.

Modelling the reservoir as an ensemble of harmonic oscillators interacting weakly with the system, it is possible to approximate the system-reservoir coupling by a linear interaction. This system can be studied by functional methods following the pioneering approach of Feynman and Vernon [8] who showed that the effect of the reservoir on the time evolution of the system is summarized in a functional, the so-called influence functional [9].

Caldeira and Leggett [4] investigated in detail the functional approach to the quantum Brownian motion showing that the reduced density matrix $\rho_{r}$ obeys, in the high-temperature limit, for an ohmic environment, a master equation which is of the following form :

$$
\frac{d \rho_{r}}{d t}=-\frac{i}{\hbar}\left[H_{R}, \rho_{r}\right]-\frac{i \gamma}{2 \hbar}\left[\{p, x\}, \rho_{r}\right]-\frac{2 m \gamma k_{B} T}{\hbar^{2}}\left[x,\left[x, \rho_{r}\right]\right]-\frac{i \gamma}{\hbar}\left(\left[x, \rho_{r} p\right]-\left[p, \rho_{r} x\right]\right)
$$

In the last equation $H_{R}$ is the renormalized self-Hamiltonian of the system, $\gamma^{-1}$ the relaxation time, $T$ the temperature. 
Zurek et al. [7] introduced the linear entropy

$$
\varsigma=\operatorname{Tr}\left(\rho_{r}-\rho_{r}{ }^{2}\right),
$$

in order to make quantitative the notion of the decoherence as measured by the vanishing of off-diagonal terms in $\rho_{r}$, which are responsible for the interference effects.

Using the master equation (Eq.(2)) for an initially pure state that remains approximately pure during the course of its evolution - a good approximation for a system with small dissipation - it is easy to show that :

$$
\begin{aligned}
\dot{\varsigma} & =4 D \operatorname{Tr}\left(\rho_{r}{ }^{2} x^{2}-\rho_{r} x \rho_{r} x\right)-2 \gamma \operatorname{Tr} \rho_{r}{ }^{2} \\
& \simeq 4 D\left(\left\langle x^{2}\right\rangle-\langle x\rangle^{2}\right)=4 D(\Delta x)^{2}
\end{aligned}
$$

where $D \equiv 2 m \gamma k_{B} T / \hbar^{2}$, and $(\Delta x)^{2}$ is the mean square deviation of the position of the system.

This defines a decoherence time scale 卅, given by :

$$
\tau_{D}=\frac{\hbar^{2}}{2 m \gamma k_{B} T(\Delta x)^{2}}
$$

which provides a measure for the amount of time required for the quantum system to start achieving classical features.

In the following section we establish our criterion for obtaining the conditions under which a macroscopic body will behave quantum mechanically.

\section{THE LIMITS}

Let us now apply the above concepts in order to obtain the quantum limits for two systems, the free mass, relevant to laser interferometric antennas, and the harmonic oscillator, relevant to mechanical bars. 


\section{A. Free Mass}

The quantum limit is obtained when $(\Delta x)^{2}$ in Eq.(4) becomes of the same magnitude as the uncertainty arising from Heisenberg's principle, when applied to successive measurement of the position of the free mass separated by a time interval $\tau$. This quantum mechanical uncertainty is given by [1]

$$
(\Delta x)^{2} \approx \frac{\hbar \tau}{m}
$$

Inserting (5) into the expression for $\tau_{D}$ ( Eq.(4)), we obtain

$$
\tau_{D}=\frac{\hbar}{2 \gamma k_{B} T \tau}
$$

If $\tau_{D}$ is greater than the time interval between two successive measurements, $\tau$, the system must be treated quantum mechanically. When this happens we obtain the quantum limit

$$
\hbar>2 \gamma k_{B} T \tau^{2}
$$

Since $\gamma^{-1}$ is the relaxation time of the system $\left(\tau^{*}\right)$, Eq. $(7)$ can be rewritten in the more usual form

$$
\hbar>2 k_{B} T \frac{\tau^{2}}{\tau^{*}}
$$

which is the limit obtained by Braginsky [3], who arrived at this result by a somewhat obscure path, as we will make explicit later on.

\section{B. Harmonic Oscillator}

For a harmonic oscillator of fundamental frequency $\omega$, the Heisenberg uncertainty principle gives 11

$$
(\Delta x)^{2} \approx \frac{\hbar}{2 m \omega}
$$


for a measurement time of the order of the period of the harmonic oscillator $\left(\tau \approx \frac{2 \pi}{\omega}\right)$. Replacing (9) into (4) leads to

$$
\tau_{D}=\frac{\hbar \omega}{\gamma k_{B} T}
$$

Imposing $\tau_{D}>\tau$ gives the quantum limit for the oscillator

$$
\hbar>\gamma \frac{k_{B} T \tau}{\omega}=\frac{k_{B} T}{\omega} \frac{\tau}{\tau^{*}}
$$

A similar result was obtained by Braginsky [3], only differing from (11) by a numerical factor.

Having shown how to obtain the quantum limits in an internally consistent way, which takes into account both quantum and thermal fluctuations, we now raise one further objection to the derivation by Braginsky of the quantum limit for a free mass.

The authors of reference [12] use as the starting point Nyquist's theorem which gives for the spectral density [** of the fluctuating force the result:

$$
\left\langle F_{f l}^{2}\right\rangle_{\omega}=4 k_{B} T \gamma
$$

Then they use this fluctuating force properly integrated over a range of frequencies $\Delta \omega \approx \tau^{-1}$ to find the displacement of the particle $x=x_{0}+\frac{1}{2} a t^{2}$ under a constant acceleration given by

$$
a=\frac{\sqrt{\left\langle F_{f l}^{2}\right\rangle_{\omega} \tau^{-1}}}{m}
$$

which then gives for the displacement

$$
\Delta x=\sqrt{\frac{k_{B} T}{m} \frac{\tau^{3}}{\tau^{*}}}
$$

This result is in disagreement with a standard treatment of the classical Brownian particle [13]. Of course the latter treatment refers to the mean square deviation of the position of the particle, which as a matter of fact is what is monitored in gravitational wave antennas [2], while Braginsky considers a displacement to be later on compared with a fluctuation (from Heisenberg's principle). 
Finally we remark that when Braginsky considers the harmonic oscillator, the classical part is treated in the right way, as a fluctuating Brownian particle in the potential of a harmonic oscillator.

\section{CONCLUSIONS}

We have obtained the conditions for the quantum behavior of a macroscopic body using a decoherence analysis, which treats the systems quantum mechanically from the very beginning, thus removing some inconsistencies present in previous treatments of the subject.

Zurek et al. showed [10] through a numerical study of the time evolution of the Wigner function that the decoherence time scale defined by Eq.(4) is still valid even at low temperatures.

It is important to stress that we do not propose the master equation (Eq.(2)) for describing the time evolution of a mechanical gravitational wave antenna for two reasons; firstly as it operates at small temperatures [2] and secondly because the dissipation mechanism for such antennas is fairly complicated [12] hardly being ohmic. We have considered in this paper a simplified model for a Weber bar and exploited some of the consequences of Eq.(2) for the quantum limits of such a system.

We are currently working on an analysis of quantum non-demolition measurements [14, taking into account dissipative effects.

\section{ACKNOWLEDGMENTS}

L.F.dos Santos and P.Marques F. would like to thank Fundação de Amparo à Pesquisa do Estado de São Paulo for financial support. C.O. Escobar wishes to thank Conselho Nacional de Desenvolvimento Científico e Tecnológico for partial financial support. 


\section{REFERENCES}

* As a matter of fact the decoherence time depends on the initial state of the system. However we always choose the smallest possible time scale for decoherence given by Eq.(4), as emphasized by Zurek et al. [10]. In so doing we are just relaxing the time condition of our decoherence criterion.

** The use of this spectral density entails a restricted limit of applicability, namely a hightemperature limit, where quantum fluctuations are completely neglected. It is unsuitable for studying the transition from classical to quantum behaviour to use it as a starting point.

[1] A. Einstein, Sitzungsber Preuss.Akad.Wiss., 688 (1916).

[2] For a review of the current status of G.W. detectors see, The detection of Gravitational Waves ed. D.G. Blair, Cambridge University Press, 1991.

[3] V.B. Braginsky and Yu.I. Vorontsov, Sov. Phys Usp. 17, 644 (1974); V.B.Braginsky, Sov. Phys. Usp. 31, 836 (1988).

[4] A.O. Caldeira and A.J. Leggett, Physica A 121, 587 (1983). W.G. Unruh and W.H. Zurek, Phys.Rev. D 40, 1071 (1989). W.H. Zurek. Phys.Today 44,No 10, 36 (1991) (for an introduction).

[5] B.L. Hu, J. P. Paz and Y. Zhang, Phys. Rev. D45, 2843 (1992); D47, 1576 (1993). M. Gell-Mann and J.B. Hartle in [6].

[6] Complexity, Entropy and the Physics of Information, ed. W. Zurek, Vol IX (AddisonWesley, Reading, 1990).

[7] W.H. Zurek, S. Habib and J.P. Paz, Phys.Rev.Lett., 70, 1187 (1993).

[8] R.P. Feynman and F.L. Vernon, Ann. Phys. 24, 118 (1963).

[9] R.P Feynman and A.R. Hibbs, Quantum Mechanics and Path Integrals, McGraw-Hill 
Book Company, 1965.

[10] J.P.Paz, S. Habib and W.H. Zurek, Phys. Rev. D47, 488 (1993).

[11] C.M. Caves, K.S. Thorne, R.W.P. Drever, V.D. Sandberg, M. Zimmermann. Rev. Mod. Phys. 52, 341 (1980).

[12] V.B. Braginsky and A.B. Manukin, Measurements of Weak Forces in Physical Experiments, Univ. of Chicago Press, 1977. V.B. Braginsky, V.P. Mitrofanov, V.I.Panov, Systems with Small Dissipation, Univ. of Chicago Press, 1985.

[13] F. Reif, Fundamentals of Statistical and Thermal Physics, McGraw-Hill, 1965.

[14] J.N. Hollenhorst, Phys.Rev. D 19,1669 (1979). 